\title{
The management of oesophageal foreign bodies and their complications
}

\author{
R. J. Donnelly \\ M.B., B.S. \\ Registrar
}

\author{
P. B. Deverall \\ F.R.C.S. \\ Senior Registrar
}

\author{
Thoracic Surgical Department, \\ General Infirmary, Leeds
}

\begin{abstract}
Summary
Five patients are described to demonstrate some of the possible clinical courses which may follow the ingestion of a foreign body. The management of such patients is discussed and evidence presented to support the case for detailed investigation, treatment and follow up.
\end{abstract}

\section{Introduction}

Many foreign bodies are swallowed, some are removed and a few cause complications. They provide a common clinical problem, especially in the edentulous and in the child, and the variety of ingested objects in terms of size, shape and consistency makes generalizations as to management difficult. This paper presents the case histories of five patients selected from a considerable number and presented as a background against which the aspects of management may be discussed.

Patients are seen because: (1) they have symptoms following swallowing, or the suspicion of swallowing, a foreign body; (2) they are anxious following the incident but are free of symptoms ; and (3) the parents of a child believe a foreign body to have been swallowed. It will, however, become apparent that each individual patient must be assessed from the point of view of possible complications. Early diagnosis is attended by improved management.

\section{Case histories}

\section{Case 1}

J.G. age 4. Ten weeks before attending hospital had a coughing and choking attack and said he had swallowed a button. He was turned upside down, slapped on the back and given bread to eat. For some days following the incident he complained of food sticking in his throat. The symptoms persisted but on an intermittent basis. When seen the main complaint was of a persistent productive cough with wheezing and on one occasion the expectoration of blood.

Oesophagoscopy performed the same day revealed a button $2 \frac{1}{2} \mathrm{~cm}$ in diameter lying transversely in the eosophagus at $15 \mathrm{~cm}$. The oesophageal mucosa was ulcerated. With some difficulty the button was removed. A gastrografin swallow was normal.

Satisfactory recovery from symptoms followed.

\section{Case 2}

H.S. age 29. Seen in the Casualty Departmen with a history of having swallowed a buttone Review of the patient's notes revealed that similar complaint had been made on previou occasions. The patient complained of slight subs sternal discomfort and a straight chest X-raæ $\overrightarrow{\mathscr{C}}$ showed a button to be at the level of the tracheaf. bifurcation.

An expectant policy was adopted with a further X-ray after $4 \mathrm{hr}$. This revealed that the button was now in the stomach and an incidental finding was four more buttons in the ileo-caecal region. The patient recovered satisfactorily.

\section{Case 3}

F.R. age 67. Three days before being seen he had swallowed a conger-eel fish bone. The patient complained of soreness in his throat. Oesophagoscopy revealed the bone just below the cricopharyngeus. The bone was removed and it was noted that the mucosa had a small tear at this site. Antibiotics were started. In the next $24 \mathrm{hr}$ the patient's condition deteriorated rapidly, with dyspnoea, cyanosis and pyrexia. A plain chest X-ray showed mediastinal widening. Cervical mediastinotomy was carried out and foul smelling blood-stained fluid found extending from the posterior pharyngeal area down into the superior mediastinum. Drainage was established but his condition continued to deteriorate and he died the following day.

Necropsy revealed extensive suppurative mediastinitis. 


\section{Case 4}

J.M. age 9. There was a previous history of oesophagitis with peptic stricture.

Two days before admission swallowed a ballbearing and following this complained of dysphagia, vomiting and lower retrosternal pain. He was taken to another hospital where a straight chest X-ray showed the foreign body in the lower oesophagus. Oesophagoscopy was performed but the ball-bearing could not be removed, an attempt therefore being made to push it on into the stomach. This was however not achieved and he was transferred for oesophagotomy to be performed. On examination at the time of admission minimal cervical surgical emphysema was noted and a further chest X-ray revealed mediastinal emphysema (Fig. 1). A gastrografin swallow confirmed the site of perforation to be at the site of the ball-bearing, on the left posterior oesophageal wall (Fig. 2). Thoractomy was performed, the ball-bearing removed and a $3 \mathrm{~cm}$ tear in the oesophagus identified, cleaned and sutured. The mediastinal tissues were opened and full drainage established. Antibiotics were given 10 days post-operatively. Oral fluids were recommended on the 10th day following a satisfactory gastrografin swallow. The child's recovery was uneventful.

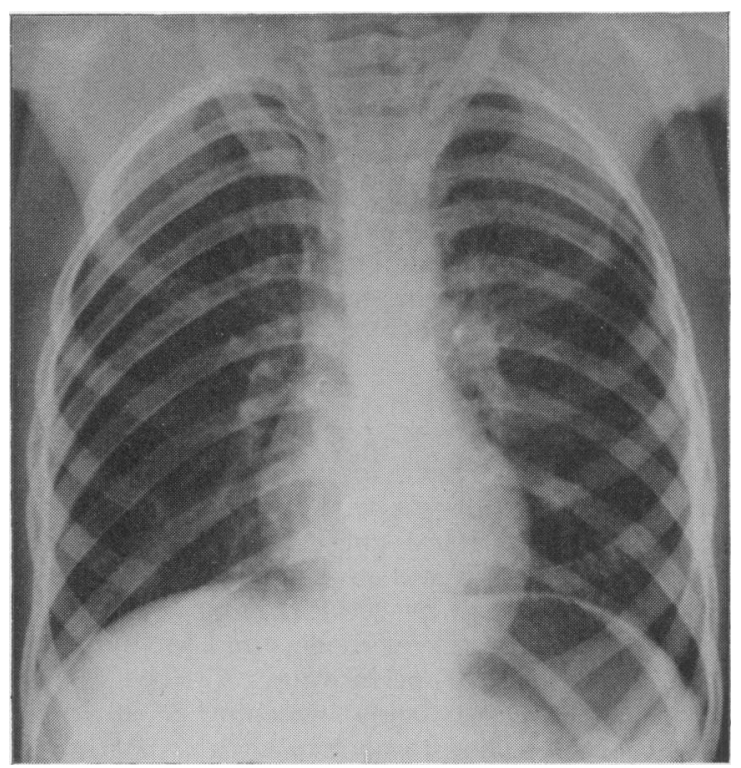

Fig. 1. Case 4. J.M. Plain chest X-ray showing mediastinal emphysema. Film taken $24 \mathrm{hr}$ after oesophagoscopy and attempted removal of a ball-bearing impacted at the lower end of the oesophagus.

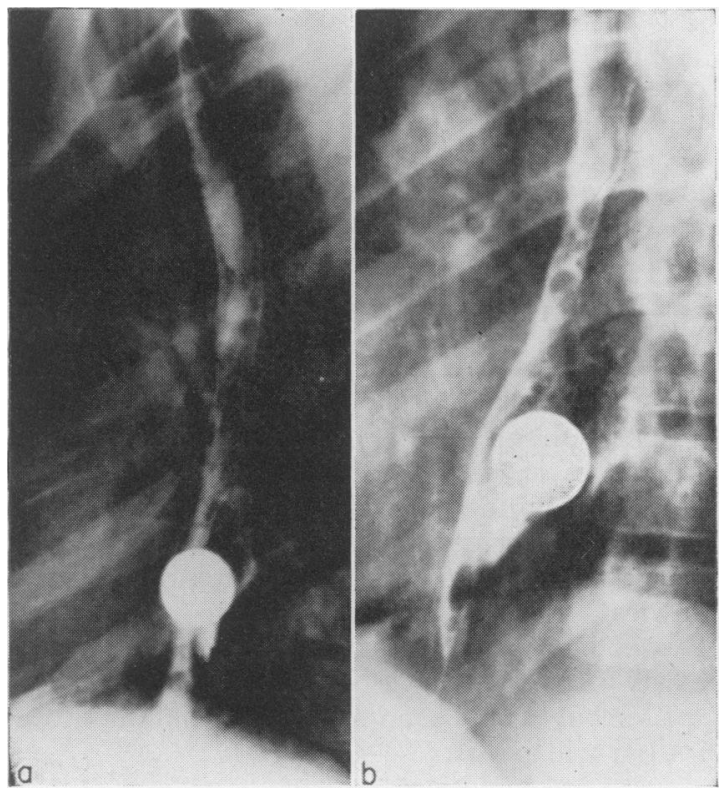

FIG. 2. Case 4. J.M. Gastrografin swallow, (a) Lateral view, demonstrating site of perforation of oesophagus. The medium can be seen escaping from the lumen in a posterior direction below the foreign body. (b) Oblique view, showing the foreign body lying to a large extent outside the oesophagus. This and other views assist in making a decision with regard to operative approach.

\section{Case 5}

D.N. age 42. Eight days before admission swallowed a piece of batter which stuck in the upper oesophagus. Soreness on swallowing persisted. Plain PA, and lateral, cervical and chest $\mathrm{X}$-rays were normal.

Oesophagoscopy revealed no foreign body but at $17 \mathrm{~cm}$ on the posterior wall the mucosa was inflamed and in one site was a linear ulcer less than $1 \mathrm{~cm}$ in length. As the area was being examined some cloudy fluid issued from the site of ulceration. Large doses of antibiotics were continued post-oesophagoscopy for 5 days and the patient reported complete relief of symptoms. A gastrografin swallow was normal. Further recovery was uneventful.

\section{Discussion}

Bisgard \& Kerr (1949) have described how the oesophageal injury resulting from a swallowed foreign body may vary from a minimal mucosal tear to a major rupture involving adjacent structures. In our experience damage to the oesophageal wall usually results from impaction, but Dawes (1964) shows clearly that perforation and mediastinitis may result from a penetrating injury 
during the onward passage of a sharp object.

Whether there has been incomplete or complete penetration of the oesophageal wall is the crucial question. Superficial inflammation, oedema, ulceration (e.g. Case 1) and intramural abscess formation (e.g. Case 5) may result from the former, whereas extramural abscess, mediastinitis and/or involvement of neighbouring structures reflect the dangers of complete penetration (e.g. Cases 3 and 4).

This clinical course following injury to the oesophagus at different levels is to some extent a reflection of the varying anatomical relations. Intrathoracic and sometimes posterior pharyngeal perforation may be attended by rapid mediastinal involvement (e.g. Case 3) (Phillips, 1938 ; Dawes, 1964), whereas anterior or lateral cervical perforation may remain localized. Dawes (1964) states that a reasonable prognosis exists if the lesion is confined to the cervical spaces but there is no guarantee that this localization will persist.

\section{Symptoms and signs}

In all patients a precise history of the type of foreign body is obtained with a description of all symptoms and their relationship in time to the incident of ingestion.

Respiratory symptoms may be the presenting feature many weeks after the original incident, especially in children (e.g. Case 1) or may be a sign of the development of mediastinitis (e.g. Case 3). Glass \& Goodman (1966) report nine cases of unsuspected oesophageal foreign body in children under the age of 3 years in whom the presenting clinical features were persistent respiratory symptoms with oesophageal symptoms is only three of the cases.

Pain, soreness on swallowing, dysphagia and/ or vomiting, and the children crying or repeated swallowing movements, are all an indication for further investigation.

Increasing soreness on swallowing and chest or back pain are of more ominous significance.

Physical signs of perforation are cervical surgical emphysema, fever, increased pulse rate, tenderness or swelling at the root of the neck and limitation of cervical movement. The crunch sound of mediastinal emphysema described by Hamman (1937) is rare and has not been elicited by us in this clinical situation. Goligher (1948), Barrett (1956) and Colman (1958) have all stressed that if perforation occurs during endoscopic removal, mediastinitis may not be apparent for $48 \mathrm{hr}$. Epigastric pain may be a presenting feature of lower oesophageal perforation if this involves the intra-abdominal oesophagus as is the case if spontaneous oesophageal rupture extends to involve this segment (Barrett, 1947 ; Moynihan, 1954 ; Barrie, 1955).

\section{Management}

Obviously many foreign bodies are swallowed and pass through the oesophagus without difficulty and generally speaking it is the presence of symptoms and signs which provide the indication for further investigation, large irregularly shaped or sharp objects being the exception in that these require location and removal even without symptoms.

The adoption of an expectant policy depends on the nature of the swallowed object in relation to the patient and the presence of associated lesions, e.g. an oesophageal web or a rigid cervical spine in an old edentulous patient, and the adoption of such a policy should be a positive action rather than a purely negative attitude of awaiting events.

We have no experience of enzymatic digestion of food boli, which was first reported by Richardson (1945) using papain (Caroid), a proteolytic enzyme which converts protein to amino acids. Further techniques have been describece by Johnson (1953) using the starch digestant Taka $\mathbb{8}$ diastase and by Miller \& Godfrey (1955) using a solution of crystalline trypsin. Robinson (1962) described three successful examples of the use of papain, given in small amounts of $5 \mathrm{ml}$ of $5 \%$ at 30-min intervals and concluded that the methoo is of value if the primary approach of oesophagoscopy is hazardous. The use of proteolytic enzyme is contra-indicated if mucosal ulceration is likely to be present, either because the bolus has been impacted for more than $24 \mathrm{hr}$ or if bone spicules are suspected to be present, and perforation of the oesophagus following the use of papain has been described by Anderson, Bernatz \& Grindley (1959).

\section{$X$-ray investigation}

We recommend $X$-ray investigation of all patients. Radio-opaque foreign bodies are identifiable in terms of shape, size and location on plain films. A lateral chest $X$-ray in the child should help to avoid overlooking an inhaled object. Mediastinal or cervical emphysema, fluid levels, pneumothorax or mediastinal widening are significant signs of perforation. A plain lateral cervical X-ray may show widening of the prevertebral space due to oedema or abscess formation and be a useful localizing sign with radiolucent objects or an early sign of posterior pharyngeal perforation.

Obviously a radiolucent object will not be 
seen on a plain film but even admitting this a negative plain film must not be accepted without reservation. Dawes (1964) comments on the cases in which perforation without impaction occurs.

In the presence of symptoms or signs and following the ingestion of large or irregularly shaped objects, a decision must be taken between endoscopy or further radiological investigation. Until recently it has been usual for oesophagoscopy to be performed at this stage as recommended by St Clair Thompson \& Negus (1955), but this approach is not supported by Pulvertaft \& Stayte (1965). They advise full radiological investigation prior to endoscopy using barium emulsion, water soluble media or barium paste. They record the example of a patient who swallowed a broken dental plate and in whom only barium paste was of positive value and they lend support to Bantam's (1965) suggestion that radioopaque material should be included in such highrisk objects, further support coming from Harvey (1965) and Bowman \& Graham (1965).

Gastrografin has the advantage of being more likely to demonstrate a small perforation, less irritating to tissues, rapidly absorbed from tissues which it has entered and less likely to cause difficulty at the time of a subsequent endoscopy. Cotton wool pledgets soaked in barium are described as being of value in demonstrating non-radio-opaque objects but we have no experience of their use.

A negative X-ray study in the presence of other features suggesting perforation does not preclude this being present, endoscopy now being essential for localization of the oesophageal damage in order to permit of correct surgical approach.

We would recommend detailed X-ray studies first if there are signs of perforation, symptoms suggesting mediastinitis, or a need, if possible, to avoid oesophagoscopy. Routine use of endoscopy is probably not justified in all hospitals, in which case unless one is prepared to risk failing to diagnose impacted non-opaque foreign bodies with their possible complications, then more detailed radiological studies should be sought.

\section{Oesophagoscopy}

There is no place for out-patient management of patients with oesophageal foreign bodies. A rounded or small object may pass on to the stomach and it is often justifiable to adopt an expectant policy. This is reasonable if the patient is seen within a few hours of the incident, is free of all but minor symptoms, and if, after a further $4 \mathrm{hr}$ there is definite evidence of the object having passed to the stomach.
Oesophagoscopy is now indicated even when there is radiological evidence of perforation as it is possible to clear the oesophagus to avoid further mediastinal soiling ; it eliminates the possbility of damage at more than one level and assists in the correct decision as regards operative approach. Dawes (1964) describes the local signs at pharyngoscopy which are suggestive of perforation at a lower oesophageal level.

Oesphagoscopy by itself is attended by a risk of perforation. Jermerin (1948), Seybould, Johnson \& Leary (1950) and Barrett (1956) consider oesophagoscopy to be the commonest causal factor in perforation. Dawes (1964) in discussing perforation found that $70 \%$ of tears in the upper oesophagus were caused by foreign bodies, while in the intrathoracic oesophagus $30 \%$ of the perforations were directly caused by the foreign body.

Goligher (1948) drew attention to posterior pharyngeal tears in older patients with a rigid cervical spine and Barrett (1956) and Colman (1958) both support his contention that this is due to pressure on the crico-pharyngeal mucosa between the oesophagoscope and the anterior surface of the cervical vertebrae.

An object which has been impacted for some time causes oesophageal damage rendering the patient at higher risk at the time of removal. Reading (1945) recorded eighty-four cases of removal of a foreign body without any case of post-operative mediastinitis. Bisgard \& Kerr (1949) however described the complication, and Dawes (1964) records a further four examples. The risks attending the failure to remove impacted foreign bodies are recorded by many authors including Neuhoff (1936), Turner (1946) and Seybould et al. (1950).

In many cases removal is accomplished and recovery is smooth. Techniques of removal are well described, particularly by Jackson \& Jackson (1948). We have no experience of the technique described by Barlow (1960) for the removal of the open safety pin. Bigler (1966) has reported the use of a balloon catheter for extracting objects impacted in the upper oesophagus. The catheter is passed distal to the object and inflated with radio-opaque medium under X-ray control, the balloon then dilating the oesophagus and pressing it away from the foreign body which becomes disimpacted and is then lifted out lying on the balloon. Bigler stresses that the technique should not be used for sharp objects or if there has been more than $24 \mathrm{hr}$ delay. It is worth recording the experience of one of the authors (P.B.D.) who lost a halfcrown during the stage of withdrawing 
oesophagoscope, forceps and foreign body. Only after some time was the coin discovered in the naso-pharynx, having caught on the soft palate.

On occasions a foreign body may pass on into the stomach as attempts are made to remove it, but the deliberate action of attempting to push an object on is dangerous (e.g. Case 4).

An impacted foreign body may be associated with a localized abscess at the site of perforation in which case the abscess may be opened into the oesophageal lumen. Case 5 is an example of an intramural abscess without a residual foreign body which was treated in this way.

\section{Surgery}

The indications for surgical intervention are:

(1) To remove a foreign body which cannot be removed through an oesophagoscope.

(2) To close a perforation of the oesophagus diagnosed within $48 \mathrm{hr}$ of injury.

(3) To drain a mediastinal abscess.

It may be impossible to remove a foreign body at oesophagoscopy either because of its size, shape or consistency or degree of impaction. In these cases thoracotomy and oesophagotomy are indicated. In addition a foreign body may penetrate the oesophageal wall and come to lie wholly or in part in the mediastinum. This will invariably be associated with a perforation and mediastinitis. Infection will be perpetuated and exaggerated by the presence of a foreign body which should, therefore, be removed. Treatment of the perforation and mediastinitis will follow the lines to be described.

Barrett (1956), referring to cases diagnosed soon after perforation, has said: 'To treat a perforation conservatively may succeed but is likely to fail in that the patient who survives the emergency may develop a mediastinal abscess or pleural complication and these result in prolonged illness and pathological sequelae which can be virtually untreatable'. We believe this is true. Seybould et al. (1950) reported a mortality of between 40 and $70 \%$ using antibiotics alone and Paulson (Paulson, Shaw \& Kee, 1960) in his series records that all four patients treated conservatively died. Foster et al. (1965) and Dawes (1964) come to a similar conclusion. The rapidly fatal course which may occur in these patients is well illustrated by Case 3. Foster et al. (1965) describe five cases in which death occurred between 2 and $22 \mathrm{hr}$ after perforation.

We suggest the following plan of management of cases complicated by perforation of the oesophagus:

\section{Patients seen within $48 \mathrm{hr}$ of perforation}

Minor anterior or lateral cervical tears may be treated conservatively with antibiotics. With these exceptions, if the site of injury can be demonstrated either radiologically or at oesophagoscopy, then the oesophagus should be explored. Dawes (1964) describes the cervical approach to the upper oesophagus. The transthoracic approach to the thoracic oesophagus should be extrapleural if possible. The foreign body should be removed if still present, the tear in the oesophagus repaired, the adjacent mediastinal tissues opened up in an attempt to prevent pocketing of infection and abscess formation, and a large drain placed to the site of perforation. It is important to remember that the mucosal tear may be more extensive than that of the muscle layer.

If it has not been possible to demonstrate the site of perforation either radiologically or at oesophagoscopy then it is impossible to plan an accurate surgical approach and such cases must be treated conservatively.

2. Patients seen more than $48 \mathrm{hr}$ after perforation

Though Poulson (Poulson et al., 1960) records the successful closure of an oesophageal teaf 27 days after the original incident, it is unlikely that the oesophageal wall will hold sutures and allow primary closure if perforation has been present for longer than $48 \mathrm{hr}$. In addition media stinitis is diffuse by this time and attempts at drainage are ineffective. Reliance must, therefore be placed on large doses of antibiotics in an endeavour to localize the infection. One or more abscesses may form and these can be drained. An abscess from an upper oesophageal injury may point in front or behind the sternomastoid muscle. The relatively simple procedure of costotransversectomy is to be preferred for drainage of an intrathoracic mediastinal abscess, but if the transthoracic approach is used every effort should be made to avoid opening the pleura.

\section{Antibiotics}

All cases require vigorous antibiotic therapy. The variety of contaminant micro-organisms which may gain entry to the mediastinum makes the choice of appropriate antibiotic difficult. For the conservative regime to be successful the correct antibiotic should be bactericidal and must reach the site of contamination in high constant concentration. In cases subjected to surgery micro-organisms are identified by culture and appropriate antibiotics prescribed, but before this information becomes available and in cases treated conservatively it is our practice to give large doses of penicillin and streptomycin. 


\section{Nutrition}

It is important in all cases to provide an adequate calorie intake either by parenteral means or by gastrostomy. Hadfield (1965) has shown the value of intravenous fat and amino acids or protein hydrolysate in relation to oesophageal healing following oesophageal resection and we believe his arguments are equally applicable to this clinical situation.

Transnasal gastric tubes are to be condemned in these patients. Not only are they irritating to the patient but they are also a hindrance to chest physiotherapy and coughing and they may cause linear ulceration of the oesophagus. Nagler \& Spiro (1963) showed that a tube across the gastro-oesophageal junction may cause reflux in the supine patient, their experiments providing a basis for the oesophageal strictures known to occur after naso-gastric intubation and discussed by Douglas (1955).

Resumption of oral intake follows the demonstration radiologically of an intact suture line in those cases treated by repair, or of healing of the perforation in those cases treated conservatively.

\section{Follow-up}

Careful follow-up is essential. Residual abscess formation is a late complication which may be asymptomatic. The penalty for omission in this respect is the slow erosion of an abscess into a vital structure or a prolonged period of ill health.

\section{Acknowledgments}

We wish to thank Mr G. H. Wooler and Mr D. A. Watson for permission to publish details of patients admitted under their care.

\section{References}

Anderson, H.A., Bernatz, P.E. \& Grindley, J.H. (1959) Perforation of the oesophagus after use of a digestive agent. Ann. Otol. (St Louis), 68, 890.

BanTAM, T.M. (1965) Dangers of radio-translucent dental plates. Brit. med. J. ii, 302.

BARLOW, D. (1960) Device for removing an open safety pin from oesophagus or bronchial tree. Lancet, i, 208.

BARRETT, N.R. (1947) Report of a case of spontaneous perforation of the oesophagus successfully treated by operation. Brit. J. Surg. 35, 216.

BARRETT, N.R. (1956) Discussion on unusual aspects of oesophageal disease. Proc. $R$. Soc. Med. 49, 529.

Bigler, F.C. (1966) The use of a Foley catheter for removal of blunt foreign bodies from the oesophagus. J. thorac. cardiovasc. Surg. 51, 759.
BISGARD, J.D. \& KERR, H.H. (1949) Surgical management of instrumental perforation of the oesophagus. Arch. Surg. 58, 739.

BORRIE, J. (1955) Spontaneous rupture of oesophagus. Brit. med. J. i, 23.

Bowman, E. \& Graham, R. (1965) Dangers of radiotranslucent dental plates. Brit. med. J. ii, 420.

Colman, B.H. (1958) Perforation of the hypopharynx and cervical oesophagus. J. Laryng. 72, 970.

DAWES, J.D.K. (1964) Traumatic perforation of the pharynx and oesophagus. $J$. Laryng. 78, 18.

Douglas, W.K. (1955) Oesophageal strictures associated with gastroduodenal intubation. Brit. J. Surg. 43, 404.

Foster, J.H., Jolly, P.C., SAWyers, J.L. \& Daniel, R.A. (1965) Oesophageal perforation. Diagnosis and treatment. Ann. Surg. 161, 701.

Glass, W.M. \& Goodman, M. (1966) Unsuspected foreign bodies in the young child's oesophagus presenting with respiratory symptoms. Laryngoscope, 76, 605.

Goligher, J.C. (1948) Postcricoid pharyngo-oesophageal perforation due to endoscopy treated by immediate suture. Lancet, i, 985.

HADField, J.I.H. (1965) Preoperative and postoperative intravenous fat therapy. Brit. J. Surg. 52, 291.

Hamman, L. (1937) Spontaneous interstitial emphysema of lungs. Trans. Ass. Amer. Phycns, 52, 311.

HaRveY, W. (1965) Dangers of radiotranslucent dental plates. Brit. med. J. ii, 420 .

JACKSON, C. \& JACKSON, C.L. (1948) Foreign bodies in air and food passages. Postgrad. Med. 4, 281.

JEMERIN, E.S. (1948) Results of treatment of perioration of oesophagus. Ann. Surg. 128, 971.

Johnson, W.M. (1953) Obstruction due to serutan. J. Amer. med. Ass. 153, 235.

Miller, J.M. \& Godfrey, G.C. (1955) Treatment of impaction of cooked meat in oesophagus with trypsin. Arch. Otolaryng. 62, 202.

Moynihan, N.H. (1954) Pressure perforation and rupture of oesophagus. Lancet, ii, 728.

NAGler, R. \& SpIRo, H.M. (1963) Persistent gastrooesophageal reflux induced during prolonged gastric intubation. New Engl. J. Med. 269, 495.

NeUHOF, H. (1936) Acute infections of mediastinum with special reference to mediastinal suppuration. $J$. thorac. Surg. 6, 184.

Poulson, D.L., Shaw, R.R. \& Kee, J.L. (1960) Recognition and treatment of oesophageal perforations. Ann. Surg. 152, 13.

PhILlips, C.E. (1938) Mediastinal infection from oesophageal perforation. J. Amer. med. Ass. 111, 998.

PulvertafT, C.N. \& STaYTe, D.J. (1965) Dangers of radiotranslucent dental plates. Brit. med. J. ii, 420.

READING, P. (1945) Some observations on aetiology of foreign bodies in oesophagus. J. Laryng. 60, 246.

RichaRDSON, J.R. (1945) New treatment for oesophageal obstruction due to meat impaction. Ann. Otol. (St Louis), $54,328$.

Robinson, A.S. (1962) Meat impaction in the oesophagus treated by enzymatic digestion.J. Amer. med. Ass. 181, 1142.

Seybold, W.D., Johnson, M.A., III \& Leary, W.V. (1950) Perforation of the oesophagus. Surg. Clin. N. Amer. 30, 1155.

St Clair Thompson \& Negus, V.E. (1955) Diseases of the Nose and Throat, p. 843. Cassell, London.

TURNer, G.G. (1946) Injuries and Diseases of the Oesophagus, p. 46. Cassell, London. 